This is an author produced version of a paper published in Theriogenology.

This paper has not been peer-reviewed and does not include the journal pagination.

Citation for the published paper:

R. Gonzalez \& Y.C.B Sjunnesson. (2013) Effect of blood plasma collected after adrenocorticotropic hormone administration during the preovulatory period in the sow on oocyte in vitro maturation. Theriogenology. Volume: 80, Number: 6, pp 673-683.

http://dx.doi.org/10.1016/j.theriogenology.2013.06.017.

Access to the published version may require journal subscription.

Published with permission from: Elsevier.

Standard set statement from the publisher:

'NOTICE: this is the author's version of a work that was accepted for publication in $<$ Theriogenology $>$. Changes resulting from the publishing process, such as peer review, editing, corrections, structural formatting, and other quality control mechanisms may not be reflected in this document. Changes may have been made to this work since it was submitted for publication. A definitive version was subsequently published in THERIOGENOLOGY, [VOL\#80 ISSUE\#6 (2013-0723)] DOI\#10.1016/j.theriogenology.2013.06.017*

Epsilon Open Archive http://epsilon.slu.se 


\title{
Effect of blood plasma collected after adrenocorticotropic hormone administration during the preovulatory period in the sow on oocyte in vitro maturation
}

\author{
Authors: R. González and, Y.C.B. Sjunnesson \\ Department of Clinical Sciences, Swedish University of Agricultural Sciences (SLU), \\ Uppsala, Sweden \\ Address: Corresponding author $\left({ }^{*}\right)$
}

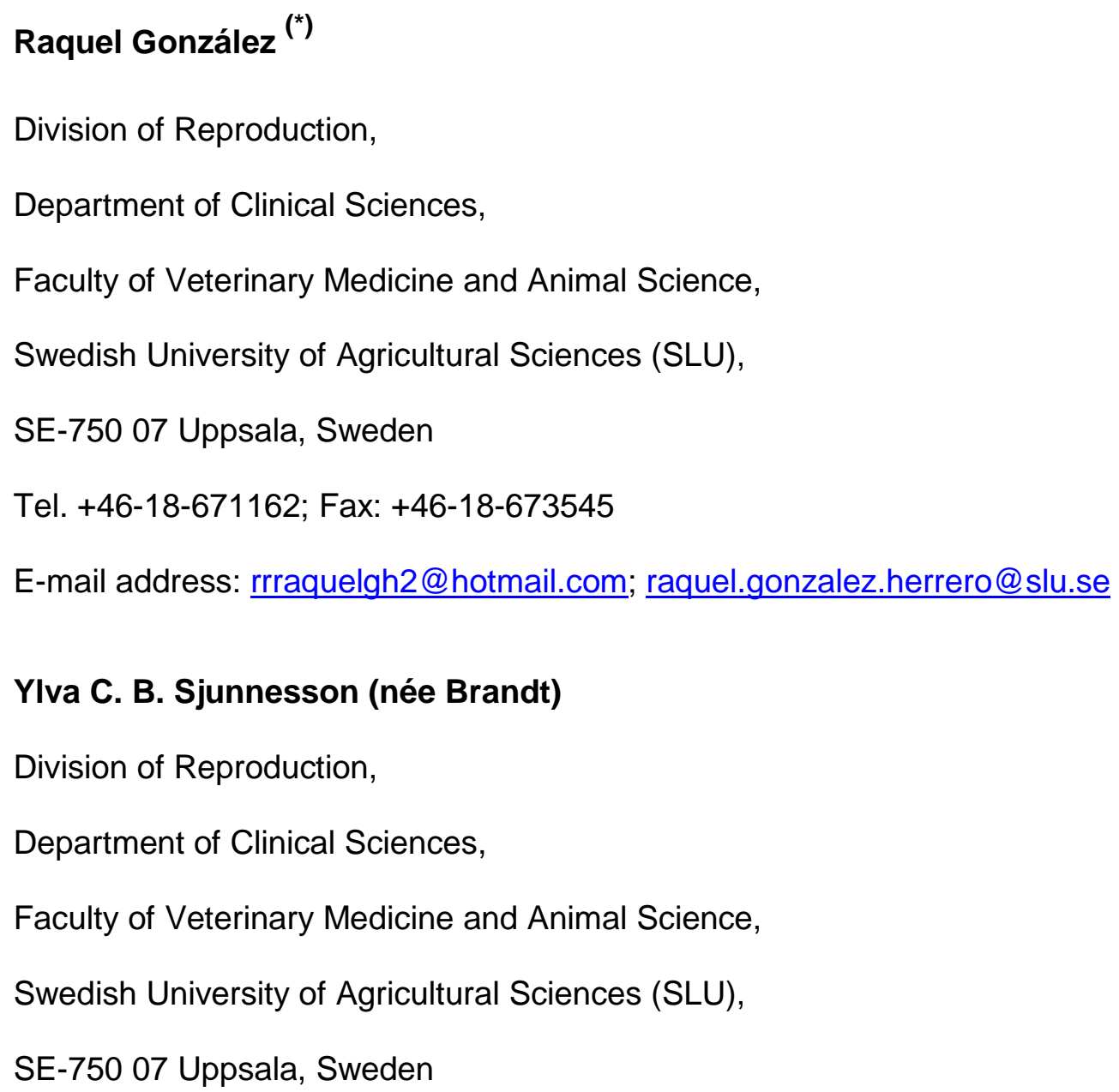


Tel. +46-18-672174; Fax: +46-18-673545

E-mail address: ylva.sjunnesson@slu.se

\section{Abstract}

Reproduction may be affected by stressful events changing the female endocrine or metabolic profile. An altered environment during oocyte development could influence the delicate process of oocyte maturation. Here, the effect of simulated stress by media supplementation with blood plasma from sows after adrenocorticotropic hormone (ACTH) administration during the preovulatory period was assessed. Oocytes were matured for $46 \mathrm{~h}$ in the presence of plasma from $\mathrm{ACTH}$-treated sows, or plasma from $\mathrm{NaCl}$-treated control sows, or medium without plasma (BSA group). The plasma used had been collected at $36 \mathrm{~h}$ and $12 \mathrm{~h}( \pm 2 \mathrm{~h}$ ) before ovulation (for the first $24 \mathrm{~h}+$ last $22 \mathrm{~h}$ of maturation, respectively). Subsequent fertilisation and embryo development were evaluated. Actin cytoskeleton and mitochondrial patterns were studied by confocal microscopy both in the oocytes and the resulting blastocysts. Nuclear maturation did not differ between treatments. Subtle differences were observed in the actin microfilaments in oocytes; however, mitochondrial patterns were associated with the treatment $(P<0.001)$. These differences in mitochondrial patterns were not reflected by in vitro outcomes, which were similar in all groups. In conclusion, an altered hormonal environment provided by a brief exposure to plasma from $\mathrm{ACTH}$-treated sows during in vitro oocyte maturation, could induce alterations in actin cytoskeleton and mitochondrial patterns in oocytes. However, these changes might not hamper the subsequent in vitro embryo development.

[217 words]

Key words: confocal microscopy / stress / porcine / actin cytoskeleton / mitochondria 


\section{Introduction}

In livestock, stress is responsible for suboptimal reproductive efficiency, which has important production and economic consequences [1]. Chronic stress is a contributing factor of reproductive failure in wild animals maintained in captivity [2] and stress of management may also disrupt the outcomes of assisted reproduction techniques in wild species $[3,4]$. Moreover, in humans, infertility has been linked with maternal psychosocial stress $[5,6]$. Recently it was shown that stress also reduced the probability of conception during the fertile window in women [7]. The changes in reproductive performance observed in stressful situations are mainly attributed to alterations in the signalling pathway at the hypothalamuspituitary axis level [8]. Glucocorticoids (GCs) released in stressful events can interfere with the secretion of gonadotrophins, but they can also affect gonadal function directly at the level of the ovary, modulating steroidogenesis or gametogenesis [8]. Therefore, the changes that GCs can cause in the hormonal profile may have an impact on the growing population of follicles or on those follicles close to ovulation. An altered follicular milieu could, in turn, result in the release of an oocyte with a compromised developmental competence $[9,10]$. However, several studies addressing the direct effects of GCs on oocyte in vitro maturation in mammals have shown conflicting results [11-14]. Previous in vivo studies in the pig model have shown that stress simulated by the administration of adrenocorticotropic hormone (ACTH) every 2 to $4 \mathrm{~h}$ during the preovulatory period, from the beginning of oestrus ( $48 \mathrm{~h}$ before ovulation) up to $\sim 12 \mathrm{~h}$ after ovulation, caused differences in the hormonal profile of treated animals and alterations in signs of sexual display $[15,16]$. Furthermore, after artificial insemination, fewer embryos were recovered in the group of $\mathrm{ACTH}$-treated sows compared to controls [17]. However, it was not possible to identify the specific step, from the preantral follicle to the early embryo stage, at which the oocytes/embryos in the simulated stress-group were lost [17]. Therefore, a possible impact of ACTH administration during the maturation of the oocyte has not been evaluated yet. 
Mitochondria are energy-converting organelles which generate ATP by oxidative phosphorylation [18] and distribute it to areas of high energy requirement. This is crucial for key events such as oocyte maturation, fertilisation and embryo development $[19,20]$. A relocation of mitochondria occurs as the oocyte matures, as shown in different species [2023]; and a disproportional inheritance of mitochondria during early cleavage can result in arrested development [24]. Therefore, mitochondrial distribution is a reliable marker of cytoplasmic oocyte maturation [25] and embryo quality [24].

The characteristics of the cytoskeleton are also useful markers to determine oocyte and embryo quality. Actin is a part of the structural cell skeleton and most development events, such as polar body release, nuclear migration and embryo cleavage, are dependent on normal actin distribution $[25,26]$. The polymerization of non-filamentous actin into microfilaments (F-actin) is important for both porcine meiosis and mitosis (i.e., for oocyte maturation and early embryo development) [27]. In addition, actin cytoskeleton integrity was previously related with embryo quality [28].

In this study, in an attempt to evaluate whether porcine oocyte maturation may be affected by an altered hormonal profile, blood plasma collected from ACTH-administered sows (simulated stress condition) during the preovulatory period was added at the equivalent points of in vitro oocyte maturation. Thus, the specific aims were to assess (i) the effect of an altered environment caused by plasma supplementation from ACTH-treated sows during in vitro maturation (IVM) of pig oocytes and (ii) their subsequent ability to undergo fertilisation and embryo development. Mitochondrial distribution and actin cytoskeleton in both oocytes and blastocysts were investigated as markers of cytoplasmic maturation and blastocyst quality, together with in vitro outcomes, and compared between the treated group and controls. 


\section{Materials and methods}

Unless otherwise indicated, all the reagents used in this study were purchased from Sigma-Aldrich, Stockholm, Sweden.

\subsection{Animals and plasma samples}

No additional in vivo experiments were performed to collect blood plasma. The plasma samples used in the present study were collected through jugular vein catheters from sows subjected to ACTH administration or control animals from a previous experiment [16]. Briefly, from the onset of standing oestrus, the sows were administered with synthetic ACTH (5 $\mu \mathrm{g} / \mathrm{kg}$ bodyweight) or $0.9 \% \mathrm{NaCl}$ every $4 \mathrm{~h}$, from the beginning of oestrus ( $\sim 48 \mathrm{~h}$ before ovulation) until $\sim 12 \mathrm{~h}$ after ovulation. Ovulation was monitored by transrectal ultrasonography. Plasma from representative sows with similar oestrous cycles was selected (determined by the LH surge, 17ß-estradiol levels and their time to ovulation) ( $n=3$; in each group) and pooled for culture supplementation during IVM (see below) to minimize individual variability. The mean plasma concentrations of cortisol and reproductive hormones are shown (Table 1). Cortisol and progesterone levels were significantly higher in the ACTH group than in the control in samples taken 45 min after the injection (Table 1) [16]. Boar husbandry was carried out as indicated [29]. The Ethics Committee for Experimentation with Animals, Uppsala, Sweden, approved the experimental design in advance. 
TTable1, Mean hormonal concentration in pooled plasma collected from ACTH-treated or control sows at different time points before ovulation.

\begin{tabular}{|c|c|c|c|c|c|}
\hline \multicolumn{6}{|c|}{ Mean hormonal concentration in plasma $36 \mathrm{~h}$ before ovulation (mean $\pm \mathrm{SD}$ ) } \\
\hline Group & $\begin{array}{l}\text { Cortisol } \\
\text { (nmol/L) }\end{array}$ & $\begin{array}{l}\text { Progesterone } \\
(\mathrm{nmol} / \mathrm{L})\end{array}$ & $\begin{array}{c}\mathrm{LH} \\
(\mu \mathrm{g} / \mathrm{L}) \\
\end{array}$ & $\begin{array}{c}\text { 17ß-estradiol } \\
(\mathrm{pmol} / \mathrm{L})\end{array}$ & $\begin{array}{l}\text { Inhibjin alpha } \\
(\mathrm{ng} / \mathrm{mL})\end{array}$ \\
\hline Control $(n=3)$ & $73.6 \pm 22.0$ & $1.3 \pm 0.5$ & $2.1 \pm 1.4$ & $52.4 \pm 9.2$ & $0.31 \pm 0.08$ \\
\hline $\mathrm{ACTH}(\mathrm{n}=3)$ & $549.5 \pm 130.9$ & $13.5 \pm 7.4$ & $3.1 \pm 1.0$ & $61.3 \pm 12.2$ & $0.34 \pm 0.04$ \\
\hline \multicolumn{6}{|c|}{ Mean hormonal concentration in plasma $12 \mathrm{~h}$ before ovulation (mean $\pm \mathrm{SD}$ ) } \\
\hline Group & $\begin{array}{l}\text { Cortisol } \\
(\mathrm{nmol} / \mathrm{L}) \\
\end{array}$ & $\begin{array}{l}\text { Progesterone } \\
(\mathrm{nmol} / \mathrm{L})\end{array}$ & $\begin{array}{c}\mathrm{LH} \\
(\mu \mathrm{g} / \mathrm{L}) \\
\end{array}$ & $\begin{array}{c}\text { 17ß-estradiol } \\
(\mathrm{pmo} / \mathrm{L})\end{array}$ & $\begin{array}{c}\text { Inhibin alpha } \\
(\mathrm{ng} / \mathrm{mL})\end{array}$ \\
\hline Control $(n=3)$ & $81.1 \pm 39.2$ & $1.1 \pm 0.1$ & $0.5 \pm 0.4$ & $5.0 \pm 1.0$ & $0.27 \pm 0.05$ \\
\hline ACTH $(n=3)$ & $546.1 \pm 79.5$ & $7.4 \pm 3.3$ & $0.9 \pm 0.6$ & $8.7 \pm 1.5$ & $0.30 \pm 0.08$ \\
\hline
\end{tabular}

ACTH was administered every $4 \mathrm{~h}$ during the preovulatory period, from the beginning of oestrus $(\sim 48 \mathrm{~h}$ before ovulation) until $\sim 12 \mathrm{~h}$ after ovulation. Monitoring of ovulation was performed by transrectal ultrasonography. Plasma collected at $36 \pm 2 \mathrm{~h}$ before ovulation was added to in vitro oocyte maturation for the first $24 \mathrm{~h}$ of culture and plasma collected at $12 \pm 2 \mathrm{~h}$ before ovulation was added for the last $22 \mathrm{~h}$ of oocyte incubation. The number of sows for plasma collection is indicated (n). For a representative statistical analysis, see [16]

\subsection{Oocyte collection and in vitro maturation}

Ovaries from prepubertal gilts were transported in $0.9 \%$ (wt/vol) sodium chloride solution at 32 to $34^{\circ} \mathrm{C}$ within 3 to 4 hours after slaughter. Cumulus oocyte complexes (COCs) were collected from 3 to $6 \mathrm{~mm}$ follicles by manual aspiration using Hepes-buffered TCM-199 (M-2520) supplemented with $2.2 \mathrm{~g} / \mathrm{L} \mathrm{NaHCO}_{3}$ (S-5761), $1 \mathrm{mM}$ L-glutamine (G-8540), 0.5 $\mathrm{mg} / \mathrm{mL}$ polyvinyl alcohol (PVA, P-8136), $20 \mathrm{IU} / \mathrm{mL}$ heparin $(\mathrm{H}-3149)$ and $50 \mu \mathrm{g} / \mathrm{mL}$ gentamicin (G-1264). The COCs were washed three times in the same collection medium but without heparin, and after selection, they were rinsed once in maturation medium. The medium used for maturation was TCM-199 (M-2154) supplemented with $50 \mathrm{ng} / \mathrm{mL}$ epidermal growth factor (E-4127), $5 \mu \mathrm{L} / \mathrm{mL}$ insulin-transferrin-selenite (ITS, I-3146), $10 \mathrm{IU} / \mathrm{mL}$ PMSG and $5 \mathrm{IU} / \mathrm{mL}$ hCG (Suigonan, Intervet Scandinavia, Skovlunde, Denmark), $1 \mathrm{mM}$ L-glutamine, $1 \mathrm{mM}$ sodium pyruvate (P-3662), $100 \mu \mathrm{M}$ 2ß-mercapto-ethanol (M-7522), and $50 \mu \mathrm{g} / \mathrm{mL}$ gentamicin. Groups of 40-55 COCs were cultured in $500 \mu \mathrm{L}$ of maturation medium into 4-well culture dishes (Nunclon, Nalgene, Nunc International, Roskilde, Denmark) for the first $24 \mathrm{~h}$ (with all the supplements) and for an additional period of $22 \mathrm{~h}$ in maturation medium without 
hormones and without ITS, at $38.5^{\circ} \mathrm{C}$ under $5 \% \mathrm{CO}_{2}$ in air. Pig oocytes were exposed to $10 \%$ pooled plasma from ACTH-treated sows ( $n=3$; ACTH group), non-treated control sows ( $n=$ 3; no ACTH group). An additional control group without plasma was included, where the medium was supplemented with $1 \mathrm{mg} / \mathrm{mL}$ BSA (A-3311) (BSA group) during in vitro maturation. The plasma added to the IVM medium for the first $24 \mathrm{~h}$ was collected around 36 $\pm 2 \mathrm{~h}$ before ovulation (to cover the period between $48-24 \mathrm{~h}$ before ovulation) and the plasma added for the last $22 \mathrm{~h}$ of culture was recovered around $12 \pm 2 \mathrm{~h}$ before ovulation (representing the period between 24-0h before ovulation). Different endpoints were evaluated after the maturation period: a) nuclear maturation: oocytes were stained to monitor progression to metaphase II; b) cytoplasmic maturation: by means of cytoskeleton morphology and actively respiring mitochondria staining in oocytes; c) ability of exposed oocytes to be fertilised and progress up to the blastocyst stage; and d) quality of the resulting blastocysts, their cytoskeletal integrity and actively respiring mitochondria. After maturation, some oocytes were stained with Hoechst $33342(\mathrm{~B}-2261 ; 10 \mu \mathrm{g} / \mathrm{mL})$ to assess their chromatin status or used for confocal laser scanning microscopy (CLSM) (6 independent replicates). An additional group of immature oocytes was included for CLSM analysis (3 replicates) to compare actin and mitochondrial patterns with mature oocytes after in vitro maturation. Degenerated oocytes were excluded from confocal analysis. For subsequent in vitro fertilisation ability and embryo development (see below) after oocyte exposure to treatments, a total of 12 replicates were carried out (Table 2).

\subsection{Semen collection and sperm preparation}

Fresh semen was collected on several occasions from one boar using the gloved-hand method. The semen was extended (1:1, v/v) in Beltsville thawing solution (BTS, 005974ZG863, IMV Technologies, L'Aigle Cedex, France), and was then washed using noncapacitating medium consisting of the following: $113.1 \mathrm{mM} \mathrm{NaCl}$ (S-5886), $4.78 \mathrm{mM} \mathrm{KCl}(\mathrm{P}-$ 
5405), $5.56 \mathrm{mM}$ D-Glucose (G-6152), $1.19 \mathrm{mM} \mathrm{KH}_{2} \mathrm{PO}_{4}(\mathrm{P}-5655), 1.19 \mathrm{mM} \mathrm{MgSO}{ }_{4} \cdot 7 \mathrm{H}_{2} \mathrm{O}(\mathrm{M}-$ 1880), $21.58 \mathrm{mM}$ sodium lactate (L-7900), $50 \mu \mathrm{g} / \mathrm{mL}$ gentamicin and $5 \mu \mathrm{g} \mathrm{mL}$ phenol red (P5530). Briefly, $0.5 \mathrm{~mL}$ of semen was transferred to a $15 \mathrm{~mL}$ tube containing $4 \mathrm{~mL}$ of noncapacitating medium at room temperature and centrifuged at $300 \mathrm{Xg}$ for $5 \mathrm{~min}$. Afterwards, the supernatant was discarded and the spermatozoa were washed again under the same conditions. The pellet was gently resuspended in IVF medium (see below), and the sperm suspension was kept at $38.5^{\circ} \mathrm{C}$ under $5 \% \mathrm{CO}_{2}$ in air for $30 \mathrm{~min}$ before being added to fertilisation wells.

\subsection{In vitro fertilisation}

After $46 \mathrm{~h}$ of maturation, oocytes were partially denuded by gently pipetting in IVF medium and rinsed twice before transferring them to a 4-well culture dish containing a final volume of $500 \mu \mathrm{L}$ IVF medium. The IVF medium composition was $90 \mathrm{mM} \mathrm{NaCl}, 12 \mathrm{mM} \mathrm{KCl}$, $0.5 \mathrm{mM} \mathrm{NaH}_{2} \mathrm{PO}_{4}(\mathrm{~S}-5011), 25 \mathrm{mM} \mathrm{NaHCO}_{3}, 0.5 \mathrm{mM} \mathrm{MgSO}_{4}, 2 \mathrm{mM}$ sodium pyruvate, $8 \mathrm{mM}$ $\mathrm{CaCl}_{2} \cdot 2 \mathrm{H}_{2} \mathrm{O}$ (C-7902), $6 \mathrm{mM}$ lactic acid (L-7900), $6 \mathrm{mg} / \mathrm{ml} \mathrm{BSA}, 1.9 \mathrm{mM}$ caffeine (C-0750), $50 \mu \mathrm{g} / \mathrm{mL}$ gentamicin and $5 \mu \mathrm{g} / \mathrm{mL}$ phenol red. The final sperm concentration used was $5 \mathrm{x}$ $10^{5}$ cells $/ \mathrm{mL}$. Gametes were co-incubated for $24 \mathrm{~h}$ at $38.5^{\circ} \mathrm{C}$ under $5 \% \mathrm{CO}_{2}$ in air.

\subsection{In vitro culture}

After gamete co-incubation, presumptive zygotes were transferred to $1.5 \mathrm{~mL}$ tubes containing $750 \mu \mathrm{L}$ NCSU-23 medium [30] supplemented with $10 \mathrm{mM}$ hepes (H-6147), 50 $\mu \mathrm{g} / \mathrm{mL}$ insulin (I-6634) and $25 \mu \mathrm{g} / \mathrm{mL}$ gentamicin to remove the remaining cumulus cells and spermatozoa using a vortex (1 min). Presumptive zygotes were then washed three times in hepes-NCSU-23 and rinsed once in porcine culture medium (PZM) [31] supplemented with $2.78 \mathrm{mM}$ myo-inositol (I-7508) and $5 \mu \mathrm{g} / \mathrm{mL}$ phenol red before placing them in $500 \mu \mathrm{L}$ PZM. After 24 hours in PZM, which corresponded with $48 \mathrm{~h}$ post-insemination (hpi), cleavage rate 
was assessed in Hepes-NCSU-23 and the non-cleaved oocytes were removed. Embryos were transferred to fresh PZM, covered with $300 \mu \mathrm{L}$ mineral oil (M-8410), and were kept in

culture up to day 7. Embryo culture was carried out in a humidified atmosphere of $5 \% \mathrm{O}_{2}, 5 \%$ $\mathrm{CO}_{2}$ and $90 \% \mathrm{~N}_{2}$. Cleavage rates were calculated as the percentage of embryos found on day 2 of culture over the total number of presumptive zygotes in culture. Blastocyst rate was calculated as the percentage of blastocysts found on day 7 of culture over the total number of embryos on day 2. Blastocyst quality was scored under a stereomicroscope as grade 1 (excellent to good) to 4 (dead or degenerating) according to the embryo grading scheme from the International Embryo Transfer Society [32]. The cytoskeleton integrity and the distribution of actively respiring mitochondria were also evaluated in the resulting blastocysts by confocal microscopy (see below).

\subsection{Simultaneous staining of mitochondria, nuclear chromatin and actin microfilaments}

For mitochondria visualization, the cell-permeant MitoTracker Orange CMTMRos probe (M-7510, Invitrogen, Stockholm, Sweden) was used, which stains mitochondria in live cells and its accumulation is dependent upon membrane potential. It is readily sequestered only by actively respiring organelles dependent upon their oxidative activity. Oocytes and blastocysts were incubated in $500 \mu \mathrm{L}$ IVM or PZM medium, respectively, with $200 \mathrm{mM}$ MitoTracker Orange at $38.5^{\circ} \mathrm{C}$ under $5 \% \mathrm{CO}_{2}$ in air for $30 \mathrm{~min}$. Afterwards, oocytes and embryos were rinsed three times in their corresponding medium and were fixed overnight at $4^{\circ} \mathrm{C}$ with $2 \%(\mathrm{w} / \mathrm{v})$ paraformaldehyde solution in PBS supplemented with $0.1 \%(\mathrm{w} / \mathrm{v})$ PVA. After fixation, samples were washed five times in PBS with 0.1\% PVA and permeabilized in 0.1\% (v/v) Triton X (X100, CAS 9002931) in PBS with PVA for $10 \mathrm{~min}$ at room temperature. Once permeabilized, the samples were incubated with Alexa Fluor ${ }^{\circledR} 488$ Phalloidin (Invitrogen, A-12379) for F-actin labelling for 1 hour at room temperature. They were washed four times (15 min per wash) in PBS with PVA at room temperature, followed by staining with 
Hoechst $33342(2.5 \mu \mathrm{g} / \mathrm{mL})$, to evaluate the maturation status of the oocyte or to perform nuclei counts on the blastocysts. Finally, the samples were rinsed three times in PBS with PVA before mounting them on diagnostic slides (Menzel gläser, Thermo Scientific, Braunschweig, Germany). For mounting, the samples were moved in $2 \mu \mathrm{L}$ of PBS and were placed in $2 \mu \mathrm{L}$ of Vectashield (Vector laboratories, Burlingame, USA), covered with a coverslip, sealed with nail polish and stored refrigerated at $4^{\circ} \mathrm{C}$ in the dark until confocal laser scanning microscopy was performed.

\subsection{Assessment of staining by confocal laser scanning microscopy (CLSM)}

Hoechst staining was evaluated under UV-fluorescence at $410 \mathrm{~nm}$. Alexa Fluor 488Phalloidin and MitoTracker Orange CMTMRos were excited by argon and helium/neon lasers at $488 \mathrm{~nm}$ and $633 \mathrm{~nm}$, respectively. Samples were analysed using a confocal laser scanning microscope (LSM 510, Carl Zeiss AB, Jena, Germany). The ZEN 2008 Light Edition Software (Carl Zeiss) was used for image analysis (http://www.zeiss.com). All pictures were analyzed together, retrospectively, at the end of the experiment to avoid changes in criteria classification throughout time. Imaging scoring was performed blind to the scorer. Two different individuals analyzed the pictures independently in duplicate for each staining.

\subsection{Classification of actin staining}

Cortical microfilaments were classified as: a) Complete: an evenly and sharply stained band of actin appearing immediately beneath the plasma membrane; b) Thin: the cortex showed a complete band of actin staining, but the density was notably lower than in the previous category and sometimes very weakly stained; c) Discontinuous: the cortical staining was interrupted and not evenly distributed throughout the periphery of the oocyte; d) Partial: when only a hemisphere of actin staining was observed beneath the oolema and; e) Absent: 
no cortical microfilaments were observed. The cytoplasmic microfilaments stained moderately and were classified in the following categories: a) Even: when a homogeneous mesh of actin staining was observed in the ooplasm; b) Not even: the actin pattern staining was heterogeneous throughout the cytoplasm and, c) Absence: no staining was observed. In addition, the presence of cumulus cells projections through the zona pellucida was identified by actin staining and classified in different patterns: a) Absence: no transzonal projections of the cumulus cells were observed; b) Intermediate presence: the presence of transzonal projections was evident, ranging from a few projections to a more abundant presence, but the stain was weak and the density of the projections was low; c) Complete presence: there was an intensely- stained network of cumulus cell processes within the whole area of the zona (Fig. 1A-F). These criteria were adapted from previous studies [33-35].
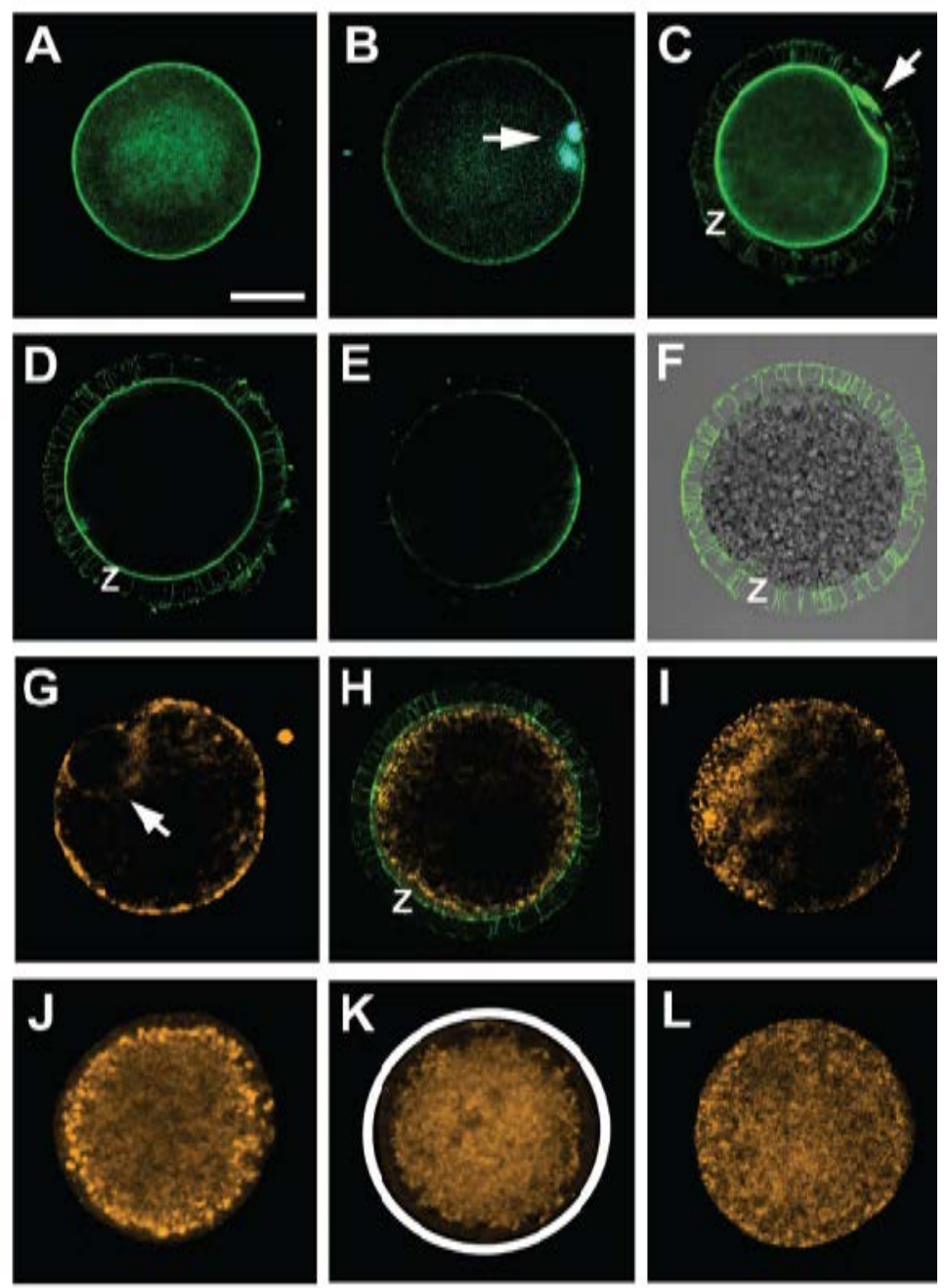
Figure 1. Representative images illustrating the different patterns of actin microfilaments (MF) and actively respiring mitochondria in mature and immature pig oocytes. Actin microfilaments are stained in green, actively respiring mitochondria in orange and chromatin in blue. (A-C, I-K) Oocytes at metaphase II (MII) and (D-H, L) at germinal vesicle (GV) stage. (A) Oocyte showing complete cortical MF, but cytoplasmic MF are not evenly distributed. (B) Mature oocyte showing decreased density of cortical and cytoplasmic MF. The MII spindle and the polar body are stained in blue (arrow). (c) Cortical and cytoplasmic MF are evenly distributed. The polar body (arrow) also shows strong actin staining and some of the cumulus cell processes within the zona pellucida (z) are evident. (D) Immature oocyte showing complete formation of cortical MF, but the density is lower than in matured oocytes. Transzonal cumulus cell projections are distinct within the zona (z). There is no apparent formation of cytoplasmic MF. (E) Oocyte showing only a partial formation of cortical microfilaments. (F) Immature oocyte in which cortical and cytoplasmic microfilaments are not formed yet. Only the cumulus cell processes within the zona pellucida (z) are evident. (G) Immature oocyte showing a peripheral distribution of actively respiring mitochondria. Some mitochondria are located surrounding the GV (arrow). (H) Oocyte in GV displaying a peripheral distribution of actively respiring mitochondria. Actin staining is also represented. Note the low density and discontinuity of cortical MF and the cumulus cell processes within the zona (z). (I) Metaphase II oocyte indicating a peripheral and polarized mitochondria distribution on the hemisphere where the spindle and the PB were located (not evident is this view). (J) Oocyte with a semiperipheral mitochondrial type after maturation. The cortex is lacking active mitochondria staining. A more intense band of actively respiring and aggregated mitochondria is evident in the subcortical area. (K) Matured oocyte with central mitochondrial pattern: the cortex does not present actively respiring mitochondria and they are located towards the central area of the ooplasm. For better identification of the cytoplasm borders, the cytoplasm is encircled in white. (L) Immature oocyte with mitochondria diffused evenly in the cytoplasm. All images are oocyte equatorial sections obtained by CLSM. Bar $=40 \mu \mathrm{m}$.

Blastocysts were classified into grades I to III according to the integrity of their actin cytoskeleton, as previously described [28, 36, 37]. Grade I was characterized by distinct actin staining in the blastomeres borders with an abundant and even microfilament complement in the cytoplasm (Fig. 3A-D). Grade /l was typified by gross maintenance of the cell outline, but with large areas where the cell borders were indistinct or stained only patchily. In addition, small clumps of actin could be seen within the cytoplasm (Fig. 3E). In grade III, the integrity of the cells was lost and the blastocysts had large areas where the cells borders were not 
distinct and microfilaments were mainly visible as clumps of actin in the cytoplasm (Fig. 3F). To classify the blastocyst into the different categories, 30 consecutive Z-stack planes were taken from each embryo. The different slices were analysed individually and after merging all scanned image layers to obtain a three-dimensional image.
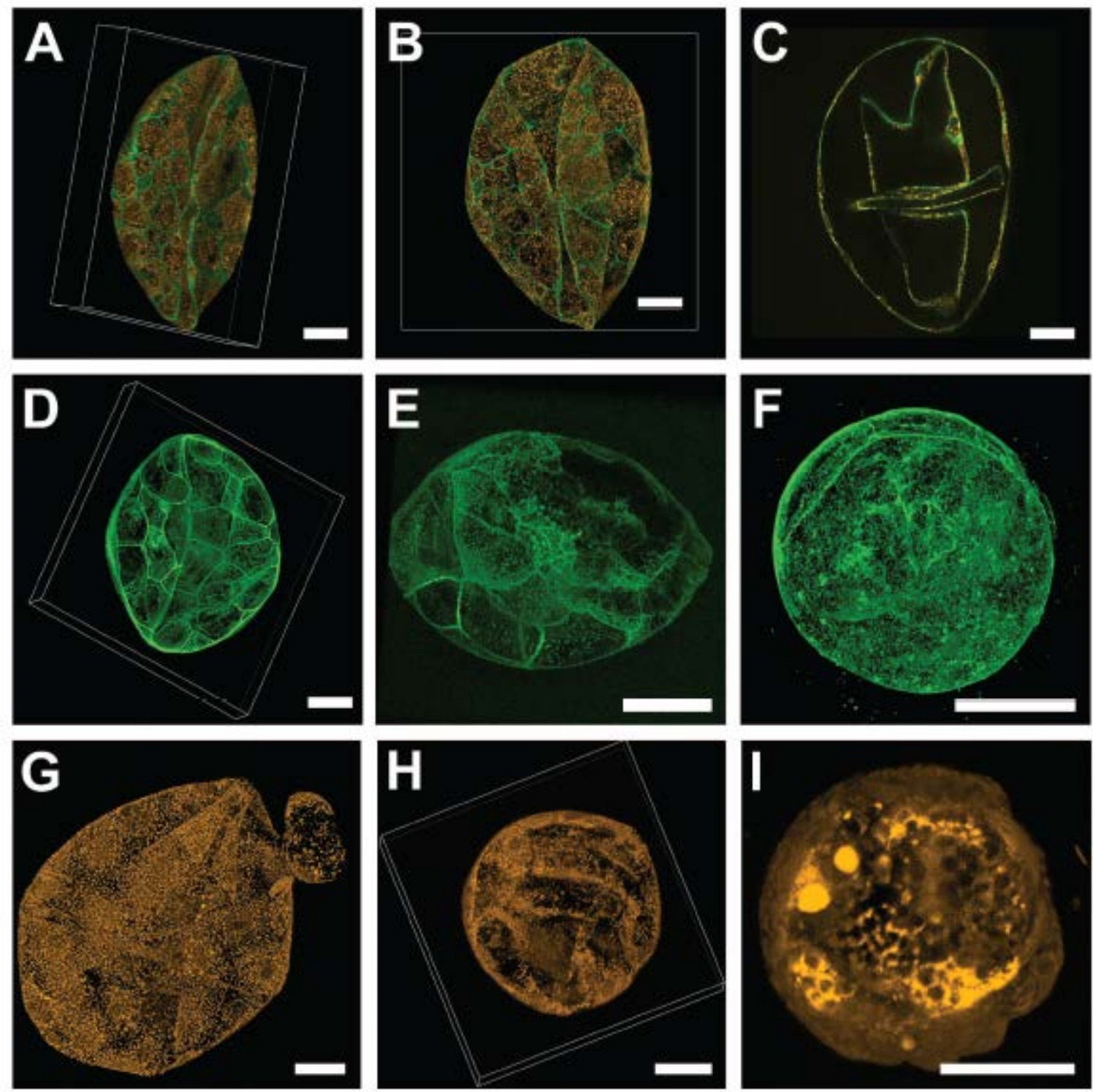

Figure 3. Representative images illustrating the different patterns of actin microfilaments (MF) and actively respiring mitochondria in pig blastocysts. Actin MF are depicted in green and actively respiring mitochondria in orange. Three-dimensional picture showing actin and mitochondria dual staining in a grade I (both for actin and mitochondria) blastocyst: (A) tilted view or (B) front view. (C) The same embryo as in A-B, but only the central slice of the Z-scan is shown. Actin cytoskeleton integrity was classified as (D) grade I, 
(E) grade II or (F) grade III. Mitochondrial pattern was categorized as (G) grade I, (H) grade II and (I) grade III. See the text for further details. White boxes are indicating the position of the embryo when tilted. Pictures without white boxes are showing the top view. Bar $=50 \mu \mathrm{m}$.

\subsection{Classification of mitochondria staining}

Oocytes were classified according to one of the following mitochondrial patterns, as defined previously [21, 23, 38]: a) Peripheral: actively respiring mitochondria were observed in the cortical region of the oocyte; b) Semi-peripheral: actively respiring mitochondria were more evident in the subcortical area whereas the cortex of the oocyte was lacking actively respiring mitochondria; c) Polarized: actively respiring_mitochondria were located mainly in one of the hemispheres of the oocyte. This pattern showed different variations, including polarization opposite to, or corresponding to the same side as, the meiotic spindle and the polar body. Oocytes showing a simultaneous peripheral and polarized distribution were included in this category; d) Central: mitochondria were localized in the centre of the cytoplasm and the periphery of the oocyte was lacking mitochondria labelling; d) Diffused: when the mitochondria were homogeneously distributed throughout the cytoplasm (Fig. 1G\llcorner) .

In blastocysts, the distribution of actively respiring mitochondria was scored from grade I to III. Grade I blastocysts had an even distribution of mitochondria where the stain was distinctly present in almost all the cells. Grade // blastocysts were characterized by an intermediate pattern, where the staining was not evenly distributed in the whole embryo; some of the blastomeres presented large clumps of staining while others lacked actively respiring mitochondria. In grade III blastocysts, the distribution of actively respiring mitochondria was very heterogeneous and $50-75 \%$ of the embryo did not present distinct points of staining (Fig. 3G-I). Z-stack (30 slices) were analysed as previously described for mitochondrial classification. 


\subsection{Statistical analysis}

Data expressed in percentages (maturation, cleavage and blastocyst rates) were analysed using one-way ANOVA. Before the analysis, normal distribution of data and residuals were tested using Shapiro-Wilk's test. Homogeneity of variances was evaluated using Levene's test. If needed, variables were arcsine-transformed to satisfy parametric assumptions. When significant differences were found, comparison between treatments was made using the post hoc Tukey test. When variables did not meet the assumptions of the parametric test (blastocyst cell number and blastocyst quality), Kruskal-Wallis ANOVA was used, followed by Mann-Whitney $U$ test to detect differences between experimental groups. The actin and mitochondrial patterns of oocytes and blastocysts were analysed as categorical data using correspondence analysis for data visualization [39]. As a complementary test to the correspondence analysis, Cramér's $\vee$ coefficient, together with Pearson chi-squared test, were calculated to measure the strength association or dependency between categorical variables [40]. Results are presented as mean \pm S.E.M, unless otherwise stated. Probability values of less than 0.05 were considered significant. Statistical analyses were performed using Statistica (Statsoft, Inc. 2001. STATISTICA, data analysis software system, version 6; www.statsoft.com). 


\section{Results}

\subsection{In vitro maturation, fertilisation and embryo culture}

In order to evaluate the effect of simulated stress on nuclear maturation, 716 oocytes were used. Some of them were used for confocal laser scanning microscopy (CLSM) assessment as indicated below. No significant differences between groups were observed in the proportion of oocytes that reached the metaphase II (MII) after $46 \mathrm{~h}$ of in vitro maturation (Table 2). For subsequent fertilisation and embryo culture, 1,333 oocytes were used. The cleavage rate, (calculated on day 2 of culture) and blastocyst rate (on day 7 of culture) did not differ between the experimental groups (Table 2).

Table 2, Results of in vitro culture (mean \pm SEM)

\begin{tabular}{|c|c|c|c|c|c|c|}
\hline & & aturation rate & & Cleavage rate & & Blastocyst rate \\
\hline Treatment & $\mathrm{n}$ & $\begin{array}{l}\% \text { (No. oocytes in } \\
\text { MIl / No. oocytes } \\
\text { cultured) }\end{array}$ & $\mathrm{n}$ & $\begin{array}{c}\% \text { (No. embryos / } \\
\text { No. presumptive } \\
\text { zygotes) }\end{array}$ & $\mathrm{n}$ & $\begin{array}{c}\% \text { (No. blastocysts / } \\
\text { No. total embryos) }\end{array}$ \\
\hline АСТН & 6 & $\begin{array}{l}63.4 \pm 4.5 \\
(145 / 226)\end{array}$ & 11 & $\begin{array}{l}54.6 \pm 3.6 \\
(218 / 405)\end{array}$ & 11 & $\begin{array}{c}29.9 \pm 3.5 \\
(67 / 218)\end{array}$ \\
\hline Control BSA & 6 & $\begin{array}{l}64.2 \pm 7.2 \\
(157 / 242)\end{array}$ & 12 & $\begin{array}{l}44.9 \pm 4.2 \\
(225 / 481)\end{array}$ & 12 & $\begin{array}{c}29.9 \pm 4.8 \\
(72 / 225)\end{array}$ \\
\hline $\begin{array}{c}\text { Control No } \\
\text { ACTH }\end{array}$ & 6 & $\begin{array}{l}63.8 \pm 2.6 \\
(158 / 248)\end{array}$ & 12 & $\begin{array}{l}51.9 \pm 3.7 \\
(231 / 447)\end{array}$ & 12 & $\begin{array}{c}27.6 \pm 2.9 \\
(65 / 231)\end{array}$ \\
\hline
\end{tabular}

Pig oocytes were exposed to pooled plasma during $46 \mathrm{~h}$ of in vitro maturation collected from ACTH treated sows, control non-ACTH treated sows or control without plasma (BSA). Maturation rate was calculated as the percentage of oocytes that reached MII after $46 \mathrm{~h}$ of maturation over the total of oocytes included in culture; cleavage rate was calculated as the percentage of embryos found on day 2 of culture over the total of presumptive zygotes in culture and blastocyst rate was calculated as the percentage of blastocysts found on day 7 of culture over the total of embryos. Statistical analysis was done using one way-ANOVA. No significant differences were found between treatments $(P>0.05)$. The number of repetitions is represented $(n)$.

Blastocyst quality assessed by the number of cells after $7 \mathrm{~d}$ of in vitro culture was similar between groups (Table 3). When blastocyst quality was scored from 1 (best quality) to 4 (worst quality), no significant differences between groups were detected if all blastocysts were included in the statistical analysis. When only expanded blastocysts (and onwards) were included in the analysis, the blastocysts obtained in the ACTH group had significantly lower quality compared to the control groups (Kruskal-Wallis test $=13.1 ; P<0.01)($ Table 3$)$. 
Table 3. Blastocyst quality (mean \pm SEM)

\begin{tabular}{ccccccc}
\hline Treatment & $\mathbf{n}$ & $\begin{array}{c}\text { Blastocyst cell } \\
\text { number }\end{array}$ & $\mathbf{n}$ & $\begin{array}{c}\text { Blastocyst } \\
\text { quality } \\
\text { (all blastocysts) }\end{array}$ & $\mathbf{n}$ & $\begin{array}{c}\text { Blastocyst quality } \\
\text { ( } \geq \text { expanded } \\
\text { blastocysts) }\end{array}$ \\
\hline ACTH & 38 & $42.6 \pm 2.4$ & 67 & $2.63 \pm 0.09$ & 35 & $2.39 \pm 0.14^{\mathrm{a}}$ \\
Control BSA & 44 & $36.2 \pm 2.1$ & 70 & $2.58 \pm 0.12$ & 26 & $1.70 \pm 0.11^{\mathrm{b}}$ \\
Control No AC TH & 36 & $42.5 \pm 3.4$ & 57 & $2.67 \pm 0.12$ & 20 & $1.73 \pm 0.15^{\mathrm{b}}$ \\
\hline
\end{tabular}

Blastocyst cell number and quality (scoring from 1 to 4 using a stereomicroscope) on day 7 of in vitro culture. Blastocysts were obtained from oocytes that were exposed to pooled plasma during $46 \mathrm{~h}$ of in vitro maturation collected from ACTH treated sows, control non-ACTH treated sows or control without plasma (BSA). Statistical analysis was done using Kruskal-Wallis ANOVA and Mann-Whitney U test. Different letters denote significant differences between treatments $(P<0.01)$. The number of analysed blastocysts is indicated $(n)$.

\subsection{Confocal laser scanning microscopy analysis in oocytes}

To assess actin cytoskeleton morphology (Figure 1), 165 oocytes were used. There were no significant differences in the cortex microfilaments in mature oocytes after treatment (ACTH, no ACTH or BSA groups) during IVM. Around 60\% of oocytes in all groups showed a complete pattern of cortex microfilaments. In contrast, only $20 \%$ of immature oocytes (noncultured) presented evenly and sharply stained cortex microfilaments $(P<0.001)$.

Cytoplasmic microfilaments differed between maturation status and treatments (Pearson chi-square: 94.7, $P<0.001$; Cramér's coefficient: 0.54). Briefly, in most of the immature oocytes (84\%), cytoplasmic microfilaments were absent and only $6 \%$ of oocytes had an even distribution of microfilaments through the cytoplasm. Among mature oocytes in the BSA group, 52\% presented an uneven pattern and $45 \%$ had an even pattern of microfilaments. However, in the plasma groups, lower numbers of oocytes presented an even distribution pattern (19\% in the ACTH group and $11 \%$ in the no ACTH group).

Regarding the transzonal cumulus cell projections, the percentage of mature oocytes without projections was observed in 41\% (control BSA), 64\% (control no ACTH) and 56\% (ACTH group). The proportion of oocytes examined after maturation with a complete 
presence of transzonal projections was $0-3.5 \%$ in all treated groups. No differences were detected between treatments $(P>0.05)$. In comparison, $98.5 \%$ of immature oocytes presented either a complete network (36\%) or various degrees of transzonal cumulus projections (62.5\%) (Pearson chi-square: 71.4; $P<0.001$; Cramér's coefficient: 0.46).

Mitochondrial staining (Figure 1) was assessed using 160 oocytes. There was an effect of the treatment during oocyte maturation on the mitochondria distribution (Pearson chisquare: 16.3; $P<0.05$; Cramér's coefficient: 0.29$)$. When immature oocytes were compared to matured oocytes, there were also differences in mitochondria distribution (Pearson chisquare: 77; $P<0.001 ;$ Cramér's coefficient: 0.40). In immature oocytes, actively respiring mitochondria were mainly observed in the periphery of the oocyte. In the BSA and ACTH group, the predominant pattern was an evenly diffused distribution of mitochondria, whereas a higher proportion of oocytes displaying a polarized pattern was found in the no ACTH group (Figure 2). 

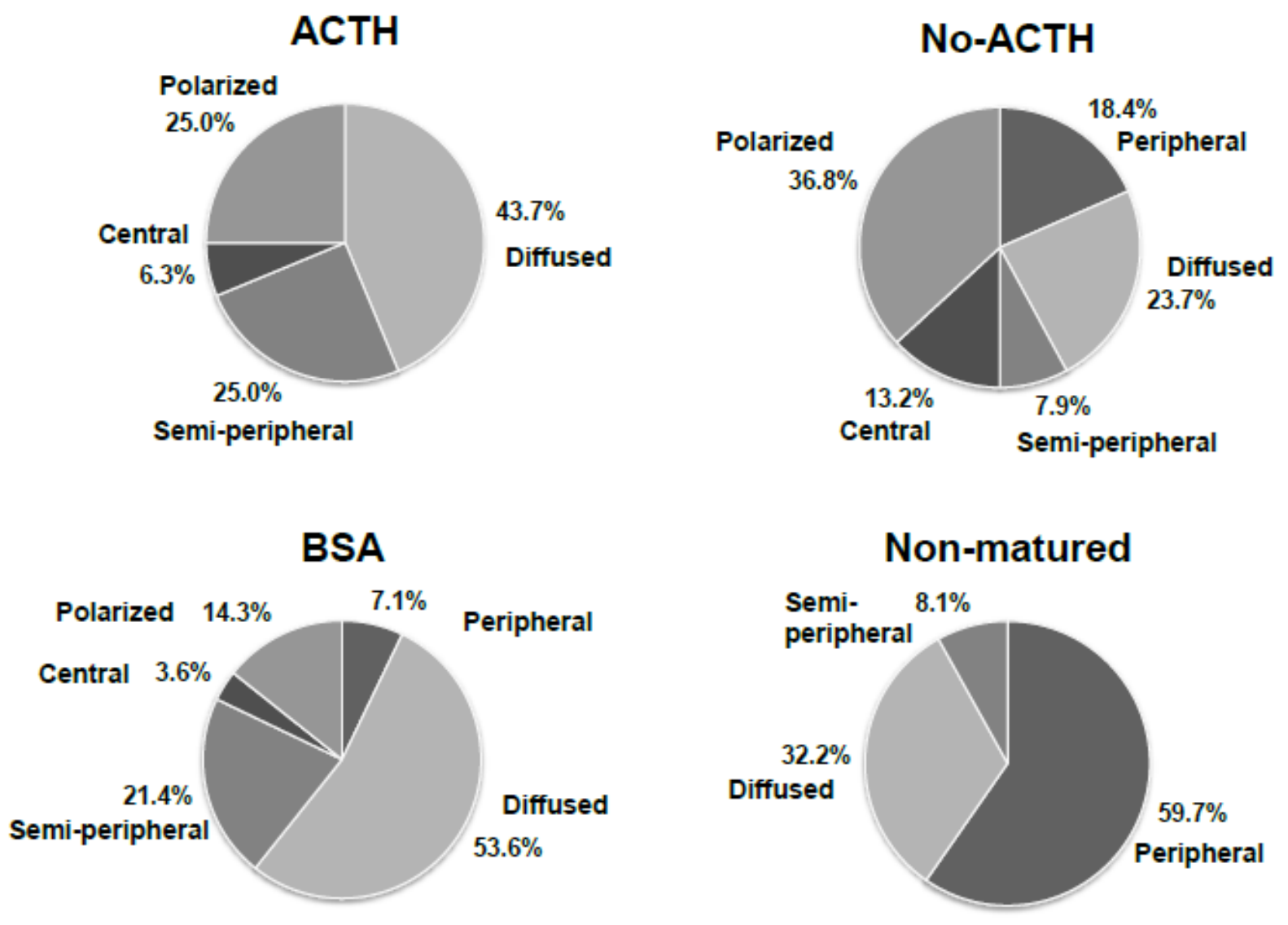

Figure 2. Distribution of mitochondrial patterns in oocytes after in vitro maturation under simulated stress conditions analysed by confocal laser scanning microscopy. Oocytes were exposed to plasma collected from ACTH stress-simulated sows (ACTH group; $n=32$ ) or belonged to different control groups: plasma collected from non-treated ACTH females (no ACTH group; $n=38$ ) or without plasma, supplemented with BSA $(n=28)$. An additional group of immature (non-matured) oocytes $(n=62)$, stained after collection was included.

\subsection{Confocal laser scanning microscopy analysis in blastocysts}

Confocal image analysis of blastocysts (Figure 3) showed that neither the actin cytoskeleton integrity nor the mitochondrial activity pattern was affected by the treatment exposure during IVM (Table 4). 
Table 4. Actin cytoskeleton integrity and mitochondrial distribution in blastocysts

\begin{tabular}{|c|c|c|c|c|c|c|c|}
\hline \multirow[b]{2}{*}{ Actin cytoskeleton integrity } & \multicolumn{3}{|c|}{ Treatment } & \multicolumn{4}{|c|}{ Statistical analysis summary } \\
\hline & $\begin{array}{c}\text { Plasma } \\
\text { ACTH }\end{array}$ & BSA & $\begin{array}{l}\text { Plasma } \\
\text { No ACTH }\end{array}$ & $\begin{array}{l}\text { Pearson Chi- } \\
\text { square }\end{array}$ & df & $P$ value & $\begin{array}{c}\text { Cramér's } \\
\text { coefficient }\end{array}$ \\
\hline Grade I & $22(53.7 \%)$ & $23(45.1 \%)$ & $27(54.0 \%)$ & 2.3 & 4 & 0.67 & 0.09 \\
\hline Grade II & $14(34.2 \%)$ & $21(41.2 \%)$ & $14(28.0 \%)$ & & & & \\
\hline Grade III & $5(12.2 \%)$ & $7(13.7 \%)$ & $9(18.0 \%)$ & & & & \\
\hline Total blastocysts & 41 & 51 & 50 & & & & \\
\hline Mitochondrial pattern & $\begin{array}{c}\text { Plasma } \\
\text { ACTH }\end{array}$ & BSA & $\begin{array}{c}\text { Plasma } \\
\text { No ACTH }\end{array}$ & $\begin{array}{l}\text { Pearson Chi- } \\
\text { square }\end{array}$ & df & p-value & $\begin{array}{l}\text { Cramér's } \\
\text { coefficient }\end{array}$ \\
\hline Grade I & $15(36.6 \%)$ & $21(42.0 \%)$ & $23(46.9 \%)$ & 2.6 & 4 & 0.63 & 0.09 \\
\hline Grade II & $19(46.3 \%)$ & $25(50.0 \%)$ & $20(40.8 \%)$ & & & & \\
\hline Grade III & $7(17.1 \%)$ & $4(8.0 \%)$ & $6(12.2 \%)$ & & & & \\
\hline Total blastocysts & 41 & 50 & 49 & & & & \\
\hline
\end{tabular}

\section{Discussion}

To tackle a possible impact of an altered hormonal environment at key events such as oocyte maturation, we have used pooled blood plasma obtained from sows subjected to ACTH administration before ovulation [16]. A similar approach was previously used [41] to study the effect of induced nutritrional hyperlipidaemia on in vitro bovine embryos; supplementing the embryo culture medium with blood plasma collected from cows under different dietary treatments. In the present study, plasma collection in relation to ovulation was known, so that the plasma recovered in vivo could be added at the corresponding time point of in vitro culture. In addition, levels of cortisol and reproductive hormones were known. Progesterone and cortisol levels were significantly higher after ACTH administration [16]. Our results indicated that, under such conditions, the exposure to plasma from ACTHadministered sows had limited impact on in vitro oocyte maturation and the subsequent ability of the oocytes to progress up to the blastocyst stage.

Glucocorticoids stimulate the production of progesterone by granulosa cells through the enzymes that regulate ovarian steroidogenesis, resulting in the combined effect of enhanced biosynthesis and decreased metabolism of progesterone (reviewed by [8]). The administration of $\mathrm{ACTH}$ raises both circulating and intrafollicular levels of cortisol [42], but the net decline of cortisol from peak concentration in plasma after ACTH administration was 
shorter in comparison to that of the decline in follicular fluid [42]. The administration of ACTH during estrous in the sow changed the plasma levels of progesterone and possibly $17 ß-$ estradiol, LH and inhibin $\alpha$ and shortened the duration of signs of standing estrous [16]. Since follicular fluids were not available, it was not possible to determine the levels of cortisol and other steroids in the follicular fluid after ACTH-administration. Thus, in the present study, plasma collected at the cortisol and progesterone peaks were selected to guarantee that plasma composition differed between the ACTH-exposed groups and controls in the assayed hormones. Blood plasma (or serum) and follicular fluid composition are similar, but contain different concentrations of hormones [43]. Nonetheless, some compounds are correlated between plasma and follicular fluid samples and variations in peripheral blood composition will be reflected in the follicular fluid [44] influencing oocyte competence. We have supplemented the in vitro maturation medium with $10 \%$ of blood plasma since it is the standard proportion used for culture media. Therefore in our work, the effect of an altered environment provided by blood plasma supplementation on oocyte maturation might have been underestimated in comparison to the environment within the follicle under ACTH exposure.

Nuclear maturation was not impaired after the exposure of oocytes to simulated stress during IVM. In agreement with other studies using GCs (cortisol, dexamethasone or corticosterone) to assess the effect of stress on the oocyte, maturation was not affected by the exposure to GCs in the mouse $[11,13]$ or sheep [12]. In addition, in the pig, a minimal inhibitory concentration of dexamethasone $(1 \mu \mathrm{g} / \mathrm{mL} \sim 2.5 \mu \mathrm{M})$ was needed to cause a negative effect on the progression of oocytes up to metaphase II, but the subsequent fertilisation ability of the oocytes was not affected [14]. This minimal inhibitory concentration of GCs is out of the physiological range, even for the levels observed after a stressful event. Cortisol is bound with high affinity to the cortisol-binding protein and also circulates bound to other proteins $[43,45]$. Around $2-5 \%$ of total endogenous cortisol corresponds to the free unbound, and thus biologically active, fraction $[43,45]$. By contrast, dexamethasone, a 
synthetic GC with similar structure to cortisol, has 25-50 times the glucocorticoid potency of cortisol and circulates mainly in the unbound form [46]. Regardless of the greater glucocorticoid potency of dexamethasone in comparison to the natural glucocorticoid, extremely high levels were needed to observe an effect on oocyte maturation in different species [12-14]. Therefore, it appears unlikely that a brief exposure to high levels of cortisol in vivo damage the oocyte directly. A recent study has evaluated the effect of maternal restraint stress on oocyte competence both in vivo and in vitro [47]. Their data suggests that restraint stress impaired oocyte competence acting through the hypothalamic-pituitaryadrenal-gonadal axes, whereas cortisol may have affected ovulation and oocyte competence by an indirect effect on the ovary rather than at the oocyte level [47]. In target tissues, cortisol can be either inactivated to cortisone or regenerated from its inactive metabolite by the bidirectional system of the 11B-hydroxisteroid dehydrogenase (HSD11B) enzymes [45]. Recently, it has been demonstrated that primarily the HSD11B enzyme system acts within porcine oocytes, converting cortisol to cortisone, and this enzymatic inactivation increases during oocyte maturation [48]. This HSD11B activity may also have limited the potentially harmful effect of physiological levels of cortisol in the oocyte in the present work. The maturation of the follicle is likely influenced by the change in estradiol:progesterone ratios within the follicle that eventually affected oocyte maturation [32]. Follicles undergoing atresia are characterized by high progesterone and low estradiol concentrations in the follicular fluid and, therefore, oocytes exposed to high levels of progesterone have lower developmental competence [32]. Nonetheless, in the present work, the high concentrations of progesterone provided by the plasma from ACTH-treated animals seemed not to limit oocyte maturation and subsequent development in comparison to their untreated counterparts. In our system, epidermal growth factor (EGF), insulin-transferrin-selenite (ITS) and 2-ßmercaptoethanol were added. 2-ßmercaptoethanol improves cytoplasmic maturation, having a beneficial effect on subsequent embryo development [49]. Both ITS and EGF also improve oocyte maturation 
in the pig $[50,51]$. Therefore, the presence of these compounds may have facilitated the ability of oocytes to overcome a possible negative effect of the altered environment.

The characteristics of actin microfilaments observed both in immature (at germinal vesicle stage) and mature (MII) oocytes were similar to those previously described in porcine oocytes [34, 35]. Minor differences were observed by CLSM in actin microfilament patterns in mature oocytes after treatment. The control group with BSA presented a greater proportion of oocytes (10-15\%) with remnants of transzonal cumulus cell projections compared with the plasma groups. This difference may be ascribed to the source of proteins, favouring cumulus expansion in the presence of plasma.

A relocation of mitochondria occurs throughout oocyte maturation $[21,38,52]$. In the pig, at the germinal vesicle stage, the majority of oocytes displayed a peripheral distribution of mitochondria, whereas at the end of IVM, mitochondria had migrated to the inner regions of the cytoplasm, a characteristic of high quality oocytes being to display a diffused homogeneous distribution of mitochondria in the cytoplasm [21, 53]. A polarization of mitochondria has been observed in MII oocytes in other animal models [22, 23], as well as in humans [54]. We have also observed polarization of mitochondria to a different extent in matured oocytes after treatment, but not in any of the immature ones. In the present study, a statistically significant effect of treatment on the mitochondria was found. Our results suggest that there was an influence of the maturation status of the oocyte, as well as a possible influence of the environment in which the oocytes are maturing on the distribution of mitochondria. In agreement with this finding, the mitochondrial distribution under in vitro conditions differed from the in vivo situation [55]; relocation of mitochondria was altered by the exposure of porcine oocytes to polychlorinated biphenyls during maturation [53]. Using multiphoton laser scanning microscopy, it was found that the pronuclear accumulation of mitochondria may be positively correlated with development to the blastocyst stage in rhesus monkey embryos [56]. In our study, the different mitochondrial distribution pattern in the various treatment groups after IVM was not reflected by cleavage or blastocyst rates. The 
oocytes were only exposed to altered hormonal levels during in vitro maturation and, therefore, the exposure time might have not been enough to detect differences at the blastocyst level. Whether the different spatial pattern of mitochondrial distribution in oocytes after maturation is related to their subsequent ability to generate blastocysts warrants future research.

Differences in blastocyst quality were observed only when expanded blastocysts (or blastocysts at later developmental stages ) were included in the analysis. However, actin cytoskeleton integrity and the general mitochondrial pattern of blastocysts were not affected by the treatment. Unfortunately, it was not possible to compare the quality score given using a stereomicroscope and the assessment by CLSM for each blastocyst, since the blastocysts were not followed individually during the staining procedure. Therefore, from our data, a detrimental effect on cytoplasmic maturation cannot be completely excluded. Whether the slight differences in blastocyst quality and maximum number of blastocyst cells reached in the treatments may be reflected in pregnancy rates and live birth rates after embryo transfer requires investigation.

In conclusion, the present work revealed that an altered hormonal environment during in vitro oocyte maturation, provided by a brief exposure to plasma collected from simulatedstressed sows by ACTH administration during the preovulatory period, could induce alterations in actin cytoskeleton and mitochondrial patterns in oocytes. However, these changes might not hamper the subsequent in vitro embryo development.

\section{Acknowledgements}

The authors would like to thank Ickholmen abattoir for providing the ovaries and Monica Nilsson for her help collecting the ovaries. We are grateful to Celina Abraham for her initial laboratory assistance and to Jane Morrell, Margareta Wallgren and Amanda Pimenta for their help with the boar semen collection. Morten Veljstedt and Anne Dorthe Roed at the Department of Basic Animal and Veterinary Sciences, Faculty of Life Sciences, University of 
Copenhagen, Denmark are gratefully acknowledged for their help with the pig IVF protocols. We are also thankful to Jane Morrell for linguistic review of this manuscript. Supported by The Swedish Research Council Formas (grant numbers 221-2007-374 and 221-2010-549). 


\section{References}

[1] Dobson H, Tebble JE, Smith RF, Ward WR. Is stress really all that important? Theriogenology. 2001;55:65-73.

[2] Swaisgood RR, Owen MA, Czekala NM, Mauroo N, Hawk K, Tang JCL. Evaluating stress and well-being in the giant panda: a system for monitoring. In: Wildt DE, Zhang A, Zhang $\mathrm{H}$, Janssen DL, Ellis S, editors. Giant pandas Biology, veterinary medicine and management: Cambridge University Press; 2006. p. 299-314.

[3] Dorn CG. Application of reproductive technologies in North American bison (Bison bison). Theriogenology. 1995;43:13-20.

[4] González R, Berlinguer F, Espeso G, Ariu F, del Olmo A, Garde JJ, et al. Use of a neuroleptic in assisted reproduction of the critically endangered Mohor gazelle (Gazella dama mhorr). Theriogenology. 2008;70:909-22.

[5] Campagne DM. Should fertilization treatment start with reducing stress? Hum Reprod. 2006;21:1651-8.

[6] Cwikel J, Gidron Y, Sheiner E. Psychological interactions with infertility among women. Eur J Obstet Gynecol Reprod Biol. 2004;117:126-31.

[7] Louis GM, Lum KJ, Sundaram R, Chen Z, Kim S, Lynch CD, et al. Stress reduces conception probabilities across the fertile window: evidence in support of relaxation. Fertil Steril. 2011;95:2184-9.

[8] Whirledge S, Cidlowski JA. Glucocorticoids, stress and fertility. Minerva Endocrinol. 2010;35:109-25.

[9] Leroy JL, Opsomer G, Van Soom A, Goovaerts IG, Bols PE. Reduced fertility in highyielding dairy cows: are the oocyte and embryo in danger? Part I. The importance of negative energy balance and altered corpus luteum function to the reduction of oocyte and embryo quality in high-yielding dairy cows. Reproduction in Domestic Animals. 2008;43:612-22. 
[10] Webb R, Garnsworthy PC, Gong JG, Armstrong DG. Control of follicular growth: local interactions and nutritional influences. J Anim Sci. 2004;83 (Suppl. E): E63-E74.

[11] Andersen CY. Effect of glucocorticoids on spontaneous and follicle-stimulating hormone induced oocyte maturation in mouse oocytes during culture. J Steroid Biochem Mol Biol. 2003;85:423-7.

[12] Gonzalez R, Ruiz-Leon Y, Gomendio M, Roldan ER. The effect of glucocorticoids on ERK-1/2 phosphorylation during maturation of lamb oocytes and their subsequent fertilization and cleavage ability in vitro. Reprod Toxicol. 2010;29:198-205.

[13] Gonzalez R, Ruiz-Leon Y, Gomendio M, Roldan ER. The effect of glucocorticoids on mouse oocyte in vitro maturation and subsequent fertilization and embryo development. Toxicol In Vitro. 2010;24:108-15.

[14] Yang JG, Chen WY, Li PS. Effects of glucocorticoids on maturation of pig oocytes and their subsequent fertilizing capacity in vitro. Biol Reprod. 1999;60:929-36.

[15] Brandt Y, Einarsson S, Ljung A, Lundeheim N, Rodríguez-Martínez H, Madej A. Effects of continuous elevated cortisol concentrations during oestrus on concentrations and patterns of progesterone, oestradiol and LH in the sow. Anim Reprod Sci. 2009;110:172-85.

[16] Brandt Y, Lundeheim N, Madej A, Rodriguez-Martinez H, Einarsson S. Effects of ACTH injections during estrus on concentrations and patterns of progesterone, estradiol, $\mathrm{LH}$, and inhibin alpha and time of ovulation in the sow. Domest Anim Endocrinol. 2007;32:122-37.

[17] Brandt Y, Madej A, Rodriguez-Martinez H, Einarsson S. Effects of exogenous ACTH during oestrus on early embryo development and oviductal transport in the sow. Reprod Domest Anim. 2007;42:118-25.

[18] Alberts B, Johnson A, Lewis J, Raff M, Roberts K, Walter P. Molecular biology of the cell. Fourth edition ed: Garland Science.; 2002. 
[19] Van Blerkom J. Mitochondria in human oogenesis and preimplantation embryogenesis: engines of metabolism, ionic regulation and developmental competence. Reproduction. 2004;128:269-80.

[20] Wang LY, Wang DH, Zou XY, Xu CM. Mitochondrial functions on oocytes and preimplantation embryos. J Zhejiang Univ Sci B. 2009;10:483-92.

[21] Brevini TA, Vassena R, Francisci C, Gandolfi F. Role of adenosine triphosphate, active mitochondria, and microtubules in the acquisition of developmental competence of parthenogenetically activated pig oocytes. Biol Reprod. 2005;72:1218-23.

[22] Nishi Y, Takeshita T, Sato K, Araki T. Change of the mitochondrial distribution in mouse ooplasm during in vitro maturation. J Nippon Med Sch. 2003;70:408-15.

[23] Velilla E, Rodriguez-Gonzalez E, Vidal F, Izquierdo D, Paramio MT. Mitochondrial organization in prepubertal goat oocytes during in vitro maturation and fertilization. Mol Reprod Dev. 2006;73:617-26.

[24] Van Blerkom J, Davis P, Alexander S. Differential mitochondrial distribution in human pronuclear embryos leads to disproportionate inheritance between blastomeres: relationship to microtubular organization, ATP content and competence. Hum Reprod. 2000;15:2621-33.

[25] Ferreira EM, Vireque AA, Adona PR, Meirelles FV, Ferriani RA, Navarro PA. Cytoplasmic maturation of bovine oocytes: structural and biochemical modifications and acquisition of developmental competence. Theriogenology. 2009;71:836-48.

[26] Sun QY, Schatten H. Regulation of dynamic events by microfilaments during oocyte maturation and fertilization. Reproduction. 2006;131:193-205.

[27] Wang WH, Abeydeera LR, Prather RS, Day BN. Actin filament distribution in blocked and developing pig embryos. Zygote. 2000;8:353-8.

[28] Zijlstra C, Kidson A, Schoevers EJ, Daemen AJJM, Tharasanit T, Kuijk EW, et al. Blastocyst morphology, actin cytoskeleton quality and chromosome content are correlated with embryo quality in the pig. Theriogenology. 2008;70:923-35. 
[29] Blomqvist G, Persson M, Wallgren M, Wallgren P, Morrell JM. Removal of virus from boar semen spiked with porcine circovirus type 2. Anim Reprod Sci. 2011;126:108-14.

[30] Petters RM, Wells KD. Culture of pig embryos. J Reprod Fertil Suppl. 1993;48:61-73.

[31] Yoshioka K, Suzuki C, Tanaka A, Anas IM, Iwamura S. Birth of piglets derived from porcine zygotes cultured in a chemically defined medium. Biol Reprod. 2002;66:112-9.

[32] Gordon I. Laboratory production of cattle embryos. Second Edition. Biotecnology in Agriculture Series No. 27 United Kingdom: CABI Publishing; 2003.

[33] Albarracin JL, Morato R, Izquierdo D, Mogas T. Effects of roscovitine on the nuclear and cytoskeletal components of calf oocytes and their subsequent development. Theriogenology. 2005;64:1740-55.

[34] Suzuki H, Jeong BS, Yang X. Dynamic changes of cumulus-oocyte cell communication during in vitro maturation of porcine oocytes. Biol Reprod. 2000;63:723-9.

[35] Suzuki H, Saito Y, Kagawa N, Yang X. In vitro fertilization and polyspermy in the pig: factors affecting fertilization rates and cytoskeletal reorganization of the oocyte. Microsc Res Tech. 2003;61:327-34.

[36] Skidmore JA, Schoevers E, Stout TA. Effect of different methods of cryopreservation on the cytoskeletal integrity of dromedary camel (Camelus dromedarius) embryos. Anim Reprod Sci. 2009;113:196-204.

[37] Tharasanit T, Colenbrander B, Stout TA. Effect of cryopreservation on the cellular integrity of equine embryos. Reproduction. 2005;129:789-98.

[38] Liu S, Li Y, Gao X, Yan JH, Chen ZJ. Changes in the distribution of mitochondria before and after in vitro maturation of human oocytes and the effect of in vitro maturation on mitochondria distribution. Fertil Steril. 2010;93:1550-5.

[39] Beh E. Simple Correspondence Analysis: A Bibliographic Review. Int Stat Rev. 2004;72:257-84.

[40] Guisande-González C, Barreiro-Felpeto A, Maneiro-Estraviz I, Riveiro-Alarcón I, Vergara-Castaño AR, Vaamonde-Liste A. Tratamiento de datos. Madrid. Spain. Ediciones Díaz de Santos; 2006. 
[41] Leroy JLMR, Van Hoeck L, Clemente M, Rizos D, Gutierrez-Adan A, Van Soom A, et al. The effect of nutritionally induced hyperlipidaemia on in vitro bovine embryo quality. Hum Reprod. 2010;25:768-78.

[42] Montgomery A, Viveiros M, Cummings E, Liptrap R. Rate of decline in cortisol concentrations in ovarian follicles following ACTH treatment in the sow. Can J Vet Res. 1997;61:309-11.

[43] Andersen CY. Possible new mechanism of cortisol action in female reproductive organs: physiological implications of the free hormone hypothesis. J Endocrinol. 2002;173:211-7.

[44] Leroy JL, Vanholder T, Delanghe JR, Opsomer G, Van Soom A, Bols PE, et al. Metabolite and ionic composition of follicular fluid from different-sized follicles and their relationship to serum concentrations in dairy cows. Anim Reprod Sci. 2004;80:201-11.

[45] Goodman HM. Basic Medical Endocrinology. Third Edition. San Diego, USA:

Academic Press; 2003.

[46] Magiakou MA, Chrousos GP. Corticosteroid therapy, non-endocrine disease and corticosteroid withdrawal. In: Bardwin C, editor. Current therapy in endocrinology and metabolism. 5th ed ed. Philadelphia: Mosby Yearbook; 1994. p. 120-4.

[47] Zhang SY, Wang JZ, Li JJ, Wei DL, Sui HS, Zhang ZH, et al. Maternal restraint stress diminishes the developmental potential of oocytes. Biol Reprod. 2011;84:672-81.

[48] Webb RJ, Sunak N, Wren L, Michael AE. Inactivation of glucocorticoids by 11betahydroxysteroid dehydrogenase enzymes increases during the meiotic maturation of porcine oocytes. Reproduction. 2008;136:725-32.

[49] Abeydeera LR, Wang WH, Cantley TC, Prather RS, Day BN. Presence of betamercaptoethanol can increase the glutathione content of pig oocytes matured in vitro and the rate of blastocyst development after in vitro fertilization. Theriogenology. 1998;50:747-56.

[50] Hu J, Ma X, Bao JC, Li W, Cheng D, Gao Z, et al. Insulin-transferrin-selenium (ITS) improves maturation of porcine oocytes in vitro. Zygote. 2011;19:191-7. 
[51] Uhm SJ, Gupta MK, Yang JH, Chung HJ, Min TS, Lee HT. Epidermal growth factor can be used in lieu of follicle-stimulating hormone for nuclear maturation of porcine oocytes in vitro. Theriogenology. 2010;73:1024-36.

[52] Sun QY, Wu GM, Lai L, Park KW, Cabot R, Cheong HT, et al. Translocation of active mitochondria during pig oocyte maturation, fertilization and early embryo development in vitro. Reproduction. 2001;122:155-63.

[53] Brevini TA, Vassena R, Paffoni A, Francisci C, Fascio U, Gandolfi F. Exposure of pig oocytes to PCBs during in vitro maturation: effects on developmental competence, cytoplasmic remodelling and communications with cumulus cells. Eur J Histochem. 2004;48:347-56.

[54] Dell'Aquila ME, Ambruosi B, De Santis T, Cho YS. Mitochondrial distribution and activity in human mature oocytes: gonadotropin-releasing hormone agonist versus antagonist for pituitary down-regulation. Fertil Steril. 2009;91:249-55.

[55] Egerszegi I, Alm H, Ratky J, Heleil B, Brussow KP, Torner H. Meiotic progression, mitochondrial features and fertilisation characteristics of porcine oocytes with different G6PDH activities. Reprod Fertil Dev. 2010;22:830-8.

[56] Squirrell JM, Schramm RD, Paprocki AM, Wokosin DL, Bavister BD. Imaging mitochondrial organization in living primate oocytes and embryos using multiphoton microscopy. Microsc Microanal. 2003;9:190-201 
